\title{
Dynamical potentials for nonequilibrium quantum many-body phases
}

\author{
Sthitadhi Roy, ${ }^{*}$ Achilleas Lazarides, Markus Heyl, and Roderich Moessner \\ Max-Planck-Institut für Physik komplexer Systeme, Nöthnitzer Straße 38, 01187 Dresden, Germany
}

(Received 23 November 2017; published 31 May 2018)

\begin{abstract}
Out of equilibrium phases of matter exhibiting order in individual eigenstates, such as many-body localized spin glasses and discrete time crystals, can be characterized by inherently dynamical quantities such as spatiotemporal correlation functions. In this paper, we introduce dynamical potentials which act as generating functions for such correlations and capture eigenstate phases and order. These potentials show formal similarities to their equilibrium counterparts, namely thermodynamic potentials. We provide three representative examples: a disordered XXZ chain showing many-body localization, a disordered Ising chain exhibiting spin-glass order, and its periodically-driven cousin exhibiting time-crystalline order.
\end{abstract}

DOI: 10.1103/PhysRevB.97.205143

\section{INTRODUCTION}

Experiments in simulators of closed quantum systems have recently observed quantum phases of inherent dynamical and nonequilibrium nature including many-body localized (MBL) [1-4] or discrete time crystal (DTC) [5-7] phases. Such phases cannot be described in terms of thermodynamic ensembles. Instead, it has been proposed that they may be characterized at the level of individual eigenstates at arbitrary energy densities leading to the notion of eigenstate phases $[8,9]$. As these phases are associated with unconventional spatiotemporal correlations, they can naturally be probed via nonequilibrium dynamics. In this paper, we develop a generally applicable framework for capturing such dynamical properties, as an alternative to the proposed single-eigenstate thermodynamics. Specifically, we introduce dynamical potentials capturing spatiotemporal correlations, characteristic of eigenstate phases. These dynamical potentials act as generating functionals for such correlations and are therefore analogous to effective potentials in the context of statistical field theory [10].

As the main focus of this paper is the introduction of the framework of dynamical potentials, it is natural to show its workings by applying it to well-studied representative example systems. As we show later in this paper, the dynamical potentials correctly characterize the out-of-equilibrium dynamics and eigenstate phases in these systems hence benchmarking the correctness of the framework. With this motivation, we study using the dynamical potentials, three examples, (i) a disordered XXZ chain, constituting the archetypal MBL system [11-21], (ii) an MBL Ising-spin glass, showing spatial eigenstate order [8,22,23], and (iii) a $\pi$-spin glass or DTC [24-27], exhibiting exotic spatiotemporal order [28,29].

The general setting we will be interested in is initializing the system in a state $\left|\psi_{0}\right\rangle$ and studying the dynamics of

\footnotetext{
*Present address: Physical and Theoretical Chemistry, Oxford University, South Parks Road, Oxford OX1 3QZ, United Kingdom and Rudolf Peierls Centre for Theoretical Physics, Clarendon Laboratory, Oxford University, Parks Road, Oxford OX1 3PU, United Kingdom.
}

an observable $\hat{\mathcal{M}}(x, t)$ under a (time-dependent) Hamiltonian $\mathcal{H}(t)$ potentially entailing eigenstate phases. These eigenstate phases can typically be detected by studying the correlations of an appropriately chosen $\hat{\mathcal{M}}$. For example, MBL can be detected by temporal persistence of finite expectation values of local operators encoding the memory of initial conditions [1,2]. Spin glass phases, on the other hand, may not be detected by such expectation values but rather via correlation functions nonlocal in space and time [28].

We construct our dynamical potential as a function of $\left|\psi_{0}\right\rangle$ and $\hat{\mathcal{M}}$ by introducing a generally space- and timedependent source field $s(x, t)$, conjugate to $\hat{\mathcal{M}}(x, t)$; successive derivatives of the constructed potential with respect to the field at $s=0$ generate the correlations of $\hat{\mathcal{M}}(x, t)$. Dynamical phases such as the MBL and Ising-spin glass can be captured via time-integrated correlations, generated by a potential corresponding to a temporally constant $s$. In this case, the first derivative yields $\int_{0}^{t} d t^{\prime} \sum_{x}\left\langle\hat{\mathcal{M}}\left(x, t^{\prime}\right)\right\rangle_{0}$, the second $\int_{0}^{t} d t^{\prime} \int_{0}^{t} d t^{\prime \prime} \sum_{x, y}\left\langle\hat{\mathcal{M}}\left(x, t^{\prime}\right) \hat{\mathcal{M}}\left(y, t^{\prime \prime}\right)\right\rangle_{0}$, and so on, where we use the notation $\langle\cdot\rangle_{0} \equiv\left\langle\psi_{0}|\cdot| \psi_{0}\right\rangle$.

Since these potentials are generating functions for manybody quantum correlations, they are associated with probability distributions whose $n$th moments give the associated $n$th order correlators. The wealth of information contained in these distributions allows us to capture various eigenstate phases. For example, a nonzero mean of the distribution reflects the temporal persistence of a nonzero expectation value of $\hat{\mathcal{M}}$, which can be used to probe nonergodicity. Similarly, a broad distribution hints towards the presence of stronger spatiotemporal correlation which betrays a spin glass.

\section{DYNAMICAL POTENTIALS}

Following ideas put forward in the context of the $s$ ensemble [30-33] we construct the generating functional as follows. For simplicity, we consider $\hat{\mathcal{M}}(x, t) \equiv \hat{\mathcal{M}}$ without any explicit space and time dependence. We couple $\hat{\mathcal{M}}$ to the system via an imaginary source field $i s(t)$ :

$$
\mathcal{H}_{s}(t)=\mathcal{H}(t)-i \frac{s(t)}{2} \hat{\mathcal{M}} .
$$


Following a nonunitary time evolution of the system with the operator $U_{t}[s]=\mathcal{T} \exp \left[-i \int_{0}^{t} d t^{\prime} \mathcal{H}_{s}\left(t^{\prime}\right)\right]$ (where $\mathcal{T}$ denotes time ordering) we define the functional

$$
\mathcal{Z}_{t}[s]=\left\langle\psi_{0}\left|U_{t}^{\dagger}[s] U_{t}[s]\right| \psi_{0}\right\rangle \equiv e^{-\Theta_{t}[s]} .
$$

An application of the Dyson equation shows that $\mathcal{Z}_{t}[s]$ is the moment generating functional (MGF) for $\hat{\mathcal{M}}(t)=$ $U_{t}^{\dagger}[0] \hat{\mathcal{M}} U_{t}[0]$. In particular the first derivative gives the expectation value $\delta \mathcal{Z}_{t}[s] /\left.\delta s(t)\right|_{s(t)=0}=\left\langle\psi_{0}|\hat{\mathcal{M}}(t)| \psi_{0}\right\rangle$ while the second gives the correlator $\delta^{2} \mathcal{Z}_{t}[s] /\left.\delta s\left(t_{1}\right) \delta s\left(t_{2}\right)\right|_{s(t)=0}=$ $\left\langle\hat{\mathcal{M}}\left(t_{1}\right) \hat{\mathcal{M}}\left(t_{2}\right)+\hat{\mathcal{M}}\left(t_{2}\right) \hat{\mathcal{M}}\left(t_{1}\right)\right\rangle_{0} / 2$ [34]. The quantity $\Theta_{t}[s]$, defined in Eq. (2), is then the associated cumulant generating functional (CGF). $\mathcal{Z}_{t}$ being the MGF, it can be recast as

$$
\mathcal{Z}_{t}[s]=\int \mathcal{D} \mathfrak{M} e^{-\int_{0}^{t} d t^{\prime} s\left(t^{\prime}\right) \mathfrak{M}\left(t^{\prime}\right)} \mathcal{P}_{t}[\mathfrak{M}],
$$

where $\mathcal{P}$ is a joint probability distribution for the temporal configuration $\mathfrak{M}(t)$. Note that $\mathfrak{M}$ is not an expectation of $\hat{\mathcal{M}}$ but rather a new classical field, defined so that functional derivatives of Eq. (3) appropriately reproduce corresponding correlations. Therefore, via Eq. (3), the quantum temporal correlations of $\hat{\mathcal{M}}$ have been encoded in the purely classical joint probability distribution $\mathcal{P}$. While $\mathcal{Z}_{t}[s]$ is easier to access numerically, inverting Eq. (3) to obtain $\mathcal{P}$ is in general nontrivial.

However, for $\Theta$ and $\mathfrak{M}$ extensive in system size $L$, the Gärtner-Ellis theorem dictates that $\mathcal{P}$ has a form $\mathcal{P}_{t}[\mathfrak{M}]=$ $e^{-L \phi_{t}[\mathfrak{M} / L]}$, thus allowing for a saddle-point approximation in the integral in Eq. (3) [35]. Defining intensive (in $L$ ) quantities $\theta=\Theta / L$ and $\mathfrak{m}=\mathfrak{M} / L$, this yields $\phi_{t}[\mathfrak{m}]$ as a Legendre transform of $\theta_{t}[s]$

$$
\phi_{t}[\mathfrak{m}]=-\max _{s}\left\{\int_{0}^{t} d t^{\prime} s\left(t^{\prime}\right) \mathfrak{m}\left(t^{\prime}\right)-\theta_{t}[s]\right\} .
$$

In two of the three examples we discuss later, it is sufficient to consider the case of a constant field $s(t)=s$. In this case, $\mathcal{Z}_{t}(s)$ and $\Theta_{t}(s)$ act as the time-integrated $\mathrm{MGF}$ and $\mathrm{CGF}$ for $\hat{\mathcal{M}}$. Explicitly, $\left.\partial_{s} \Theta_{t}\right|_{s=0}=\int_{0}^{t} d t^{\prime}\left\langle\hat{\mathcal{M}}\left(t^{\prime}\right)\right\rangle_{0} \equiv \mathcal{A} \quad$ and $\left.\quad \partial_{s}^{2} \Theta_{t}(s)\right|_{s=0}=$ $\int_{0}^{t} d t_{1} \int_{0}^{t} d t_{2}\left(\left\langle\hat{\mathcal{M}}\left(t_{1}\right) \hat{\mathcal{M}}\left(t_{2}\right)\right\rangle_{0}-\left\langle\hat{\mathcal{M}}\left(t_{1}\right)\right\rangle\left\langle\hat{\mathcal{M}}\left(t_{2}\right)\right\rangle_{0}\right) \equiv \mathcal{X}$. Accordingly, Eq. (3) becomes

$$
\mathcal{Z}_{t}(s)=e^{-L \theta_{t}(s)}=\int d \mu e^{-L s \mu} P_{t}(\mu, L),
$$

with $P_{t}(\mu, L)=e^{-L \phi_{t}(\mu)}$ and $\phi_{t}(\mu)=-\max _{s}\left[s \mu-\theta_{t}(s)\right]$, analogously to Eq. (4), where $\mu$ is an intensive in $L$ variable. Hence, $\phi_{t}(\mu)$ and $\theta_{t}(s)$ are related to each other formally in a fashion similar to that of thermodynamic potentials. The moments of $P_{t}(\mu)$ correctly reproduce the time-integrated temporal correlations as $\int d \mu \mu^{n} P_{t}(\mu, L)=\left.L^{-n} \partial_{s}^{n} \mathcal{Z}_{t}(s)\right|_{s=0}$. In particular,

$$
\operatorname{mean}\left[P_{t}(\mu, L)\right]=\mathcal{A} / L ; \operatorname{var}\left[P_{t}(\mu, L)\right]=\mathcal{X} / L^{2} .
$$

The validity of a saddle point approximation in Eq. (5) relies on the variance of $P_{t}(\mu, L)$ decreasing with increasing $L$, which translates onto a condition on $\mathcal{X}$ that it must scale at most as $L^{2}$.

We now use the framework to study eigenstate phases in three representative examples.
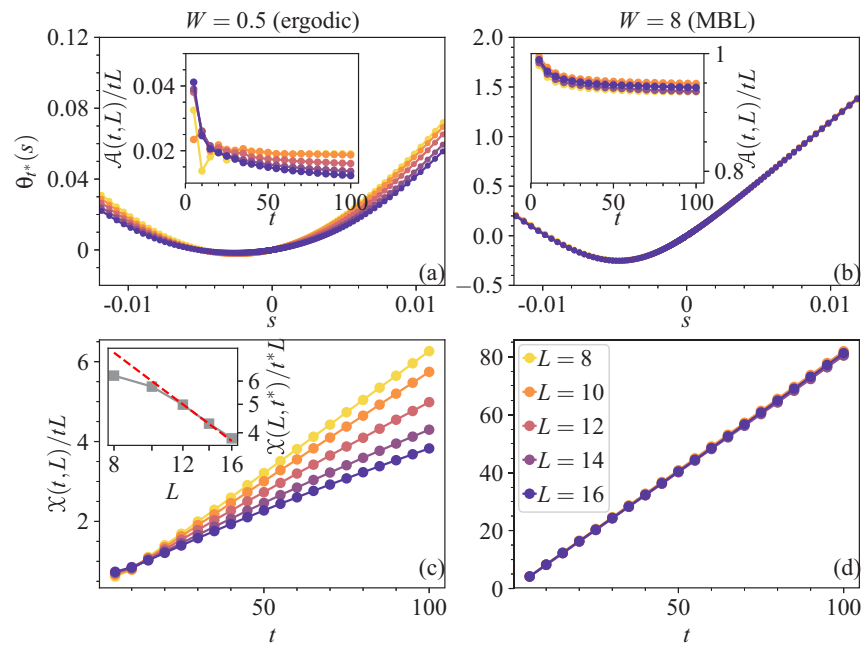

FIG. 1. The potential $\theta_{t}(s)$ for the disordered XXZ chain: (a),(b) $\theta(s, t)$ for fixed $t=t^{*}=100$ for different values of $L$, indicating that as $L \rightarrow \infty, \theta$ approaches a fixed function. The inset shows the first derivative $\left.\partial_{s} \theta_{t}(s)\right|_{s=0}, \mathcal{A}(t, L) / L$ divided by $t$ corresponding the time-averaged value of the Neél order parameter, which shows a vanishingly small value in the ergodic phase, whereas a persistent finite value in the MBL phase. (c),(d) The second derivative, $\mathcal{X}(L, t) / L$ divided by $t$, suggesting a scaling $\mathcal{X}(t, L) / L \sim t^{2} L^{-1}$ and $t^{2} L^{0}$ in the ergodic and MBL phases, respectively. The red dashed line in the inset of (c) corresponds to $L^{-1}$. Other parameters are $J=1$ and $J_{z}=0.3$, and the data is averaged over $\approx 500$ disorder realizations.

\section{DISORDERED XXZ CHAIN}

We start with the archetypal model for MBL, the random field spin-1/2 XXZ chain [14,15,18]:

$$
\mathcal{H}_{\mathrm{XXZ}}=\sum_{l}\left[J\left(\sigma_{l}^{x} \sigma_{l+1}^{x}+\sigma_{l}^{y} \sigma_{l+1}^{y}\right)+J_{z} \sigma_{l}^{z} \sigma_{l+1}^{z}+h_{l} \sigma_{l}^{z}\right],
$$

where, $\sigma_{l}^{\alpha}$ s denote the Pauli matrices for the spin- $1 / 2$ at site $l$, and the random fields $h_{l}$ are drawn from a uniform distribution $[-W, W]$. For $W>W_{c}$ with $W_{c} / J \approx 3.5$ the system resides in an MBL phase while for $W<W_{c}$ it is ergodic [18] at energy densities corresponding to infinite temperature. The two phases have been characterized, both theoretically [36] and experimentally [1], by the dynamics of the staggered magnetization starting from an initial Neél state $\left|\psi_{0}\right\rangle=|\uparrow \downarrow \uparrow \downarrow \ldots\rangle$ motivating our choice of $\hat{\mathcal{M}}=\sum_{l}(-1)^{l} \sigma_{l}^{z}$ and $\left|\psi_{0}\right\rangle$. In the ergodic phase, $\langle\hat{\mathcal{M}}(t)\rangle_{0} \rightarrow 0$ for $t \rightarrow \infty$ whereas $\langle\hat{\mathcal{M}}(t)\rangle_{0} \neq 0$ for all $t$ in the MBL phase.

Our results for $\theta_{t}(s)$ are shown in Fig. 1 comparing the ergodic (left column) and MBL (right column) phases. $\theta_{t}(s)$ for a fixed $t$ plotted against $s$ in Figs. 1(a) and 1(b) collapses for different $L$ in the MBL phase, whereas in the ergodic phase a systematic system size dependence is present. The properties of $\theta_{t}(s)$ are explored in more detail by studying $\mathcal{A}$ and $\mathcal{X}$ defined above. The time-averaged staggered magnetization density given by $\mathcal{A} / t L$ tends to zero with increasing $L$ in the ergodic phase, consistent with the expectation that local spatial information is washed out in the long-time limit. In the MBL phase on the other hand, $\mathcal{A} / t L \neq 0$ for long times with 

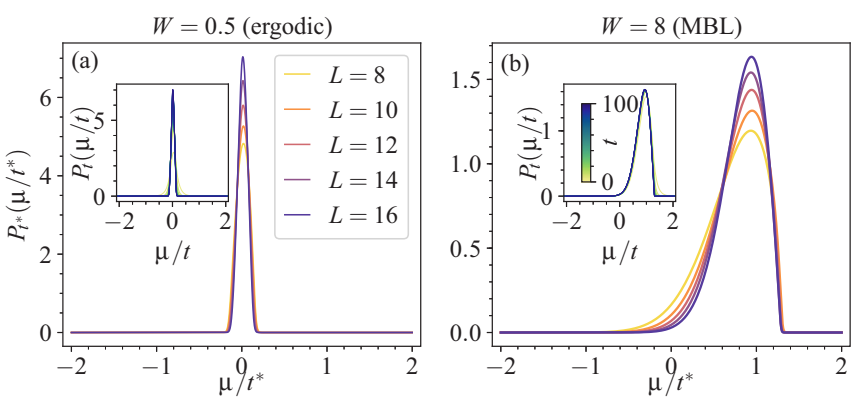

FIG. 2. Probability distribution $P_{t}(\mu / t)$ for the disordered XXZ chain: In the ergodic phase (a), the distribution is peaked around zero whereas in the MBL phase (b), it is around a finite value. Due to the presence of persistent temporal correlations in the MBL phase, the distribution is visibly wider compared to the ergodic phase where they are absent. The insets show the distributions for $L=16$ and different values of $t$ showing that $P_{t}(\mu / t)$ becomes time independent for large times. This is therefore the infinite-time result.

a very weak and unsystematic system size dependence, which we attribute to finite size effects. The behavior of $\mathcal{A} / t L$ in the two phases is shown in the insets of Figs. 1(a) and 1(b).

The difference between the MBL and ergodic phases also manifests itself in the temporal quantum correlations contained in $\mathcal{X}$. Figure $1(\mathrm{~d})$ shows $\mathcal{X} / t L \sim t$ indicating strong longrange temporal correlations in the MBL phase, and the absence of any scaling with $L$ implies the temporal correlations persist in the thermodynamic limit. By contrast, the ergodic phase has temporal quantum correlations decreasing with system size like $\mathcal{X} / t L \sim t L^{-1}$ as shown in Fig. 1(c), thus vanishing in the thermodynamic limit, consistent with the ergodic nature of the system.

The scalings of $\mathcal{X}$ show that it scales at most linearly with $L$ and satisfies the criteria [mentioned below Eq. (6)] for the applicability of the saddle-point approximation in Eq. (5). $\phi_{t}(\mu)$ and consequently $P_{t}(\mu)$ can thus be obtained from Legendre transforming $\theta_{t}(\mu)$. In Fig. 2 we show results for $P_{t}(\mu / t)$ [37] obtained this way for the same data as in Fig. 1. For a fixed $L, P_{t}(\mu / t)$ for different times collapse onto each other indicating that they have converged to the infinite-time result. While the distribution has a peak at zero in the ergodic phase, in the MBL phase the peak is at a finite value of $\mu / t$ reflecting a vanishing and finite time-averaged expectation value in the ergodic and MBL phases, respectively. The variances $\operatorname{var}\left[P_{t}(\mu / t)\right]$ scale as $\sim L^{-2}$ and $L^{-1}$ in the ergodic and MBL phases, respectively. Since the temporal correlation $\mathcal{X}$ scales the same way as $t^{2} L^{2} \operatorname{var}\left[P_{t}(\mu / t)\right]$, the scalings of $\operatorname{var}\left[P_{t}(\mu / t)\right]$ in the two phases in principle implies the temporal persistence of correlations in the thermodynamic limit in the MBL phases and their absence in the ergodic phase.

\section{DISORDERED ISING CHAIN}

Our second example is a disordered Ising chain, exhibiting an MBL spin glass-paramagnet transition, described by the Hamiltonian

$$
\mathcal{H}_{\mathrm{ISG}}=\sum_{l}\left[J_{l} \sigma_{l}^{x} \sigma_{l+1}^{x}+J_{z} \sigma_{l}^{z} \sigma_{l+1}^{z}+h_{l} \sigma_{l}^{z}\right],
$$
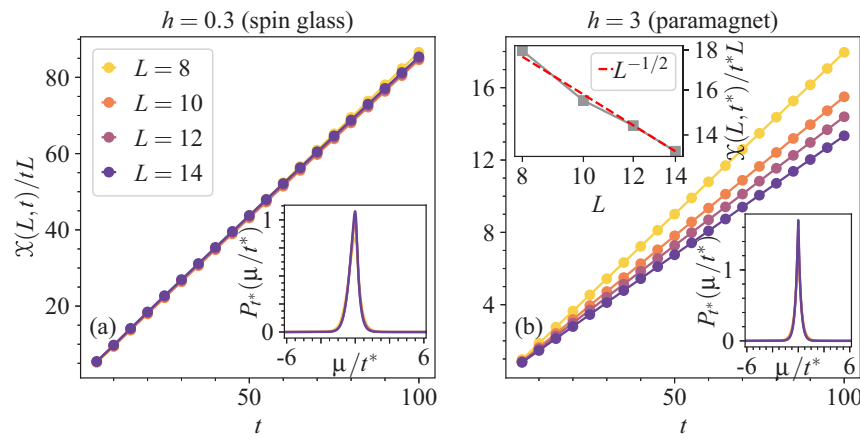

FIG. 3. Results for the disordered Ising chain: The second derivative of $\theta$ with respect to $s$ at $s=0$ is shown for the (a) spin glass and (b) paramagnet phase which suggests the scaling of the form $\mathcal{X} / L \sim$ $t^{2} L^{0}$ and $t^{2} L^{-1 / 2}$, respectively. The corresponding distributions $P_{t}(\mu / t)$ are shown in the insets. The upper inset in (b) confirms the scaling form of $\mathcal{X}(t, L)$ with $L$. Other parameters are $J=1, J_{z}=0.3$, $\Delta J=5$, and $W=0.5$.

where $J_{l} \in[-\Delta J, \Delta J]$ and $h_{l}=h+\Delta h_{l}$ with $\Delta h_{l} \in$ $[-W, W]$. For sufficiently large $\Delta J$ and weak $h$ and $W$, the system is in an MBL spin-glass phase displaying localizationprotected order and in a paramagnetic phase otherwise [8]. In particular, the Edwards-Anderson order parameter density, $O_{\mathrm{EA}}=\sum_{\alpha} \sum_{l>m}\left\langle\alpha\left|\sigma_{l}^{x} \sigma_{m}^{x}\right| \alpha\right\rangle^{2} /\left(2^{L} L^{2}\right)$ is finite in the spinglass phase and vanishes in the paramagnetic phase; here $|\alpha\rangle$ denotes the eigenstates of $\mathcal{H}_{\text {ISG }}[8,22,23]$.

To apply the framework of dynamical potentials to this example, we choose the operator $\hat{\mathcal{M}}=\sum_{l>m} \sigma_{l}^{x} \sigma_{m}^{x} / \sqrt{L}$. The rationale behind the choice is twofold. Firstly, the infinite-time averaged two-time correlator of this operator, $\quad \lim _{t \rightarrow \infty} t^{-2} \int_{0}^{t} d t_{1} \int_{0}^{t} d t_{2}\left\langle\hat{\mathcal{M}}\left(t_{1}\right) \hat{\mathcal{M}}\left(t_{2}\right)\right\rangle_{0}=$ $\sum_{\alpha}\left|\left\langle\psi_{0} \mid \alpha\right\rangle\right|^{2} \mathcal{M}_{\alpha \alpha}^{2}$, is the Edwards-Anderson order parameter, $O_{\text {EA }}$, provided the initial state is an infinite temperature state [38]. We therefore perform our numerical calculations using random product states as initial states. Secondly, this choice of operator leads to the dynamical potential $\Theta_{t}(s)$ being extensive in $L$, so that the saddle-point approximation in Eq. (5) is valid. Note that the first moment, $\lim _{t \rightarrow \infty} t^{-1} \int_{0}^{t} d t\langle\hat{\mathcal{M}}(t)\rangle_{0}=\sum_{\alpha}\left|\left\langle\psi_{0} \mid \alpha\right\rangle\right|^{2} \mathcal{M}_{\alpha \alpha}$, vanishes in both phases. It is only the second moment that distinguishes between them, as it reproduces the EA order parameter as described above.

Figure 3 shows our numerical results for $\mathcal{X}$. In Figs. 3(a) and 3(b) $\mathcal{X} / L \sim t^{2} L^{0}$ and $\sim t^{2} L^{-1 / 2}$, respectively; since $\lim _{t \rightarrow \infty} \mathcal{X} / t^{2} L=O_{\mathrm{EA}}$, we conclude that the system is in the spin-glass phase for panel (a) and in the paramagnetic phase for panel (b).

The associated probability distributions $P_{t}(\mu)$ are shown as insets in Fig. 3 where not only the width of the distribution is parametrically suppressed in the paramagnet, we also observe a fundamental difference in their shapes depending on the phase in which the system resides. The variances $\operatorname{var}\left[P_{t}(\mu)\right]$ scales differently in the two phases, $\sim t^{2} L^{1}$ and $\sim t^{2} L^{3 / 2}$ in the spin-glass and paramagnet, respectively. More interestingly, unlike that for spin glass, $P_{t}(\mu)$ for the paramagnet appears to become nonanalytic at its peak. This follows from the fact that in this phase and in the thermodynamic limit the leading 
term in $\theta_{t}(s)$ is $\sim s^{4}$ (since $\mathcal{X}$ vanishes) so that its Legendre transform $\phi_{t}(\mu) \sim|\mu|^{3 / 4}$ around $\mu=0$.

\section{FLOQUET DISCRETE TIME CRYSTAL- $\pi$-SPIN GLASS PHASE}

Our third example system hosts a phase with exotic spatiotemporal order, namely the $\pi$-spin glass or DTC phase exclusive to Floquet systems [24-26]. This example involves an explicitly time-dependent $\mathcal{H}$ and $s$, demonstrating the applicability of our framework for this type of a system.

The Hamiltonian for this model is again the disordered Ising Hamiltonian of Eq. (8) with parameters periodically modulated in time according to $J(t)=J(1+\operatorname{sgn}[\sin (\Omega t)]) / 2$ and $h(t)=h(1-\operatorname{sgn}[\sin (\Omega t)]) / 2$, with $\Omega=2 \pi / T$ denoting the frequency. In this case it has been shown that there exists an extended region of the two-dimensional parameter space of $h / \Omega$ and $J / \Omega$ where every Floquet eigenstate and its parity-reversed partner are separated by quasienergy $\Omega / 2=$ $\pi / T$ with $T$ the period so the phase was termed the $\pi$-spin glass, while simultaneously exhibiting spin-glass order [24]. This structure results in the expectation values of certain local observables, for instance, local longitudinal magnetizations, exhibiting a periodicity with frequency $\Omega / 2$ or period $2 T$. This motivates the terminology "discrete time crystal," as the observables break the discrete temporal translation symmetry of the underlying Hamiltonian by time $T$ to a lower symmetry, namely, translation by $2 T$.

Since temporal order is the hallmark of the DTC, we build the dynamical potentials using a time-dependent probe field $s(t)=s \cos (\omega t)$ coupled to $\hat{\mathcal{M}}=\sum_{l} \sigma_{l}^{x}$ :

$$
\mathcal{H}_{s}(\omega, t)=\mathcal{H}_{\mathrm{DTC}}(t)-i \frac{s}{2} \cos (\omega t) \hat{\mathcal{M}} .
$$

For simplicity, let us consider the fully polarized initial state $\left|\psi_{0}\right\rangle=\otimes_{l}|+\rangle_{l}$ with $\sigma_{l}^{x}|+\rangle_{l}=|+\rangle_{l}$ and $\hat{\mathcal{M}}$ as above. For a general initial product state, one instead needs to consider the operator $\hat{\mathcal{M}}=\sum_{l} \sigma_{l}^{x}\left\langle\sigma_{l}^{x}\right\rangle_{0}$ to take into account the nontrivial Edwards-Anderson order parameter.

We calculate the frequency dependent response of the system $\mathcal{A}(t, \omega)=\left.\partial_{s} \Theta_{t}(s)\right|_{s=0}$. For $\Omega / J=2 \pi$ and $h / J=\pi / 2$, the system is known to be deep inside the $\pi$-spin glass phase. The results are presented in Fig. 4(a), which shows that the response grows linearly with $t$ for $\omega=\Omega / 2$ and is vanishingly small otherwise. This is a direct signature of $\langle\hat{\mathcal{M}}(t)\rangle_{0}$ persistently oscillating at $\omega=\Omega / 2$ and hence of the time-crystalline order. To study the behavior away from $h / J=\pi / 2$, we calculate $\tilde{\mathcal{A}}(\omega, h)=\lim _{t \rightarrow \infty} \mathcal{A}(t, \omega, h) / t$ as a function of $h$ and find that the phase is stable over an extended range of $h$, see Fig. 4(b).

The appearance of time crystalline order in local observables relies on the existence of spatial spin glass order protected by localization, so as to stop the system from heating up to infinite temperature [39-41]. The dynamical potentials offer the possibility of simultaneously studying the spatial and temporal order. For simplicity let us consider the initial state and $\hat{\mathcal{M}}$ to be as above. The second derivative of the dynamical potential becomes $\mathcal{X}=$ $\int d t_{1} \int d t_{2} \sum_{i, j}\left\langle\sigma_{i}^{x}\left(t_{1}\right) \sigma_{j}^{x}\left(t_{2}\right)\right\rangle_{c} \cos \left(\omega t_{1}\right) \cos \left(\omega t_{2}\right)$, where $\langle\cdot\rangle_{c}$ denotes a connected correlator. In the $\pi$-spin glass phase,
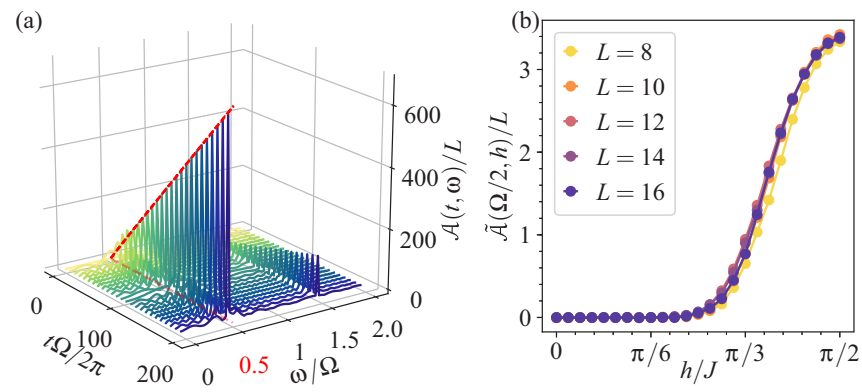

FIG. 4. Results for the DTC: (a) For $h=\pi / 2$, i.e., in the $\pi$-spin glass phase, the system shows a persistent response growing with time only when probed at $\omega=\Omega / 2$ indicating the time-crystalline order. This is also highlighted by the red line. (b) The analog of the order parameter for the $\pi$-spin glass phase, $\tilde{\mathcal{A}}(\Omega / 2, h)$ decays with $h$ continuously to zero indicating the phase transition out of the phase.

(i) the spatial correlation function in the integrand may be nonvanishing due to the spatial spin-glass order, while (ii) the correlator is periodic in $t_{1}-t_{2}$ with frequency $\Omega / 2$, probing the temporal order. Since the integral picks out the $\omega$-frequency component of the correlator, it acts as a probe for combined spatiotemporal order at the frequency $\omega$. The spin glass in the previous example corresponds to the case of $\omega=0$ as the spin-glass order is static and hence a time-independent $s$ is sufficient. Thus, the dynamical potentials can be used to characterize spatiotemporal order in a unified fashion by incorporating $\omega$ as a free parameter.

\section{OUTLOOK}

In this paper, we take the first step towards a general framework, analogous to statistical mechanics, for studying nonequilibrium closed quantum systems, focusing on eigenstate phases in such systems. We do so by constructing dynamical potentials which encode spatiotemporal correlations central to characterizing eigenstate phases such as MBL spin glasses and DTCs. It is interesting to note that the framework obtains classical probability distributions arbitrary moments of which can generate spatiotemporal correlations of arbitrary orders. However, for the sake of showing the workings of the framework, we only considered examples where the correlations of first and second orders suffice for complete understanding of the eigenstate order.

Of particular future interest is the application of the framework to study eigenstate phase transitions which might be reflected in the full distributions. Using the formal similarity between dynamical and thermodynamic potentials to study universality of such transitions seems very appealing as a future step. It appears as a promising approach to extend and use methods known in equilibrium statistical physics, for instance field theoretic techniques or renormalization group methods. In fact, since the analog of partition function here is nothing but the expectation value of identity calculated over two time-evolved states, one could use methods from Keldysh Green's functions and then treat the non-Gaussian part of the Hamiltonians perturbatively using diagrammatic or other methods to obtain (semi)analytic but approximate answers in the thermodynamic limit. 
Furthermore, in the examples we studied, the nonequilibrium phases could be detected by the dynamics of local observables or spatial few-point correlations. To what extent the formalism can be generalized to study nonlocal string order parameters relevant for out-of-equilibrium topological phases [42-45] is an open question, as is the applicability of the framework to spatiotemporally nonlocal correlations quantifying the dynamics of information spreading such as outof-time ordered correlations [46]. Finally, using the framework to study intermediate-time features of the dynamics, such as prethermalization plateaux [47-49], remains a subject of future research.

\section{ACKNOWLEDGMENTS}

We acknowledge valuable discussions with J. P. Garrahan. This work was supported by the Deutsche Forschungsgemeinschaft via the Gottfried Wilhelm Leibniz Prize program.
[1] M. Schreiber, S. S. Hodgman, P. Bordia, H. P. Lüschen, M. H. Fischer, R. Vosk, E. Altman, U. Schneider, and I. Bloch, Observation of many-body localization of interacting fermions in a quasirandom optical lattice, Science 349, 842 (2015).

[2] J. Smith, A. Lee, P. Richerme, B. Neyenhuis, P. W. Hess, P. Hauke, M. Heyl, D. A. Huse, and C. Monroe, Many-body localization in a quantum simulator with programmable random disorder, Nat. Phys. 12, 907 (2016).

[3] J.-y. Choi, S. Hild, J. Zeiher, P. Schauß, A. Rubio-Abadal, T. Yefsah, V. Khemani, D. A. Huse, I. Bloch, and C. Gross, Exploring the many-body localization transition in two dimensions, Science 352, 1547 (2016).

[4] P. Bordia, H. Lüschen, U. Schneider, M. Knap, and I. Bloch, Periodically driving a many-body localized quantum system, Nat. Phys. 13, 460 (2017).

[5] J. Zhang, P. W. Hess, A. Kyprianidis, P. Becker, A. Lee, J. Smith, G. Pagano, I.-D. Potirniche, A. C. Potter, A. Vishwanath et al., Observation of a discrete time crystal, Nature (London) 543, 217 (2017).

[6] S. Choi, J. Choi, R. Landig, G. Kucsko, H. Zhou, J. Isoya, F. Jelezko, S. Onoda, H. Sumiya, V. Khemani et al., Observation of discrete time-crystalline order in a disordered dipolar manybody system, Nature (London) 543, 221 (2017).

[7] S. Pal, N. Nishad, T. S. Mahesh, and G. J. Sreejith, Temporal Order in Periodically Driven Spins in Star-Shaped Clusters, Phys. Rev. Lett. 120, 180602 (2018).

[8] D. A. Huse, R. Nandkishore, V. Oganesyan, A. Pal, and S. L. Sondhi, Localization-protected quantum order, Phys. Rev. B 88, 014206 (2013).

[9] R. Nandkishore and D. A. Huse, Many-body localization and thermalization in quantum statistical mechanics, Annu. Rev. Condens. Matter Phys. 6, 15 (2015).

[10] G. Mussardo, Statistical Field Theory: An Introduction to Exactly Solved Models in Statistical Physics (Oxford University Press, Oxford, 2010).

[11] I. V. Gornyi, A. D. Mirlin, and D. G. Polyakov, Interacting Electrons in Disordered Wires: Anderson Localization and Low$T$ transport, Phys. Rev. Lett. 95, 206603 (2005).

[12] D. M. Basko, I. L. Aleiner, and B. L. Altshuler, Metalinsulator transition in a weakly interacting many-electron system with localized single-particle states, Ann. Phys. 321, 1126 (2006).

[13] V. Oganesyan and D. A. Huse, Localization of interacting fermions at high temperature, Phys. Rev. B 75, 155111 (2007).

[14] M. Žnidarič, T. Prosen, and P. Prelovšek, Many-body localization in the Heisenberg XXZ magnet in a random field, Phys. Rev. B 77, 064426 (2008).
[15] A. Pal and D. A. Huse, Many-body localization phase transition, Phys. Rev. B 82, 174411 (2010).

[16] R. Vosk and E. Altman, Many-Body Localization in One Dimension as a Dynamical Renormalization Group Fixed Point, Phys. Rev. Lett. 110, 067204 (2013).

[17] D. A. Huse, R. Nandkishore, and V. Oganesyan, Phenomenology of fully many-body-localized systems, Phys. Rev. B 90, 174202 (2014).

[18] D. J. Luitz, N. Laflorencie, and F. Alet, Many-body localization edge in the random-field Heisenberg chain, Phys. Rev. B 91, 081103 (2015).

[19] R. Vosk, D. A. Huse, and E. Altman, Theory of the Many-Body Localization Transition in One-Dimensional Systems, Phys. Rev. X 5, 031032 (2015).

[20] E. Altman and R. Vosk, Universal dynamics and renormalization in many-body-localized systems, Annu. Rev. Condens. Matter Phys. 6, 383 (2015).

[21] D. A. Abanin and Z. Papić, Recent progress in many-body localization, Ann. der Phys. 529, 1700169 (2017).

[22] D. Pekker, G. Refael, E. Altman, E. Demler, and V. Oganesyan, Hilbert-Glass Transition: New Universality of TemperatureTuned Many-Body Dynamical Quantum Criticality, Phys. Rev. X 4, 011052 (2014).

[23] J. A. Kjäll, J. H. Bardarson, and F. Pollmann, Many-Body Localization in a Disordered Quantum Ising Chain, Phys. Rev. Lett. 113, 107204 (2014).

[24] V. Khemani, A. Lazarides, R. Moessner, and S. L. Sondhi, Phase Structure of Driven Quantum Systems, Phys. Rev. Lett. 116 250401 (2016).

[25] D. V. Else, B. Bauer, and C. Nayak, Floquet Time Crystals, Phys. Rev. Lett. 117, 090402 (2016).

[26] N. Y. Yao, A. C. Potter, I.-D. Potirniche, and A. Vishwanath, Discrete Time Crystals: Rigidity, Criticality, and Realizations, Phys. Rev. Lett. 118, 030401 (2017).

[27] W. W. Ho, S. Choi, M. D. Lukin, and D. A. Abanin, Critical Time Crystals in Dipolar Systems, Phys. Rev. Lett. 119, 010602 (2017).

[28] C. W. von Keyserlingk, V. Khemani, and S. L. Sondhi, Absolute stability and spatiotemporal long-range order in Floquet systems, Phys. Rev. B 94, 085112 (2016).

[29] R. Moessner and S. L. Sondhi, Equilibration and order in quantum Floquet matter, Nat. Phys. 13, 424 (2017).

[30] J. P. Garrahan, R. L. Jack, V. Lecomte, E. Pitard, K. van Duijvendijk, and F. van Wijland, Dynamical First-Order Phase Transition in Kinetically Constrained Models of Glasses, Phys. Rev. Lett. 98, 195702 (2007). 
[31] L. O. Hedges, R. L. Jack, J. P. Garrahan, and D. Chandler, Dynamic order-disorder in atomistic models of structural glass formers, Science 323, 1309 (2009).

[32] J. P. Garrahan and I. Lesanovsky, Thermodynamics of Quantum Jump Trajectories, Phys. Rev. Lett. 104, 160601 (2010).

[33] J. M. Hickey, S. Genway, I. Lesanovsky, and J. P. Garrahan, Time-integrated observables as order parameters for full counting statistics transitions in closed quantum systems, Phys. Rev. B 87, 184303 (2013).

[34] See Supplemental Material (Sec. I) at http://link.aps.org/ supplemental/10.1103/PhysRevB.97.205143 for the derivation and results for the $n$th derivative.

[35] H. Touchette, The large deviation approach to statistical mechanics, Phys. Rep. 478, 1 (2009).

[36] D. J. Luitz, N. Laflorencie, and F. Alet, Extended slow dynamical regime close to the many-body localization transition, Phys. Rev. B 93, 060201 (2016).

[37] Since $\int d \mu \mu P_{t}(\mu)=\int_{0}^{t} d t^{\prime}\left\langle\hat{\mathcal{M}}\left(t^{\prime}\right)\right\rangle_{0}$, if the variable $\mu$ is not scaled with $t$, the peak position of the distribution $P_{t}(\mu)$ shifts with $t$ in the MBL phase while it would settle at zero in the ergodic phases. See Fig. A1 in the Supplemental Material at http://link.aps.org/supplemental/10.1103/PhysRevB.97. 205143.

[38] See Supplemental Material (Sec. II) at http://link.aps.org/ supplemental/10.1103/PhysRevB.97.205143 for details of the choice of order parameter for the Ising spin glass.

[39] A. Lazarides, A. Das, and R. Moessner, Equilibrium states of generic quantum systems subject to periodic driving, Phys. Rev. E 90, 012110 (2014).
[40] A. Lazarides, A. Das, and R. Moessner, Fate of Many-Body Localization Under Periodic Driving, Phys. Rev. Lett. 115, 030402 (2015).

[41] P. Ponte, Z. Papić, F. Huveneers, and D. A. Abanin, Many-Body Localization in Periodically Driven Systems, Phys. Rev. Lett. 114, 140401 (2015).

[42] C. W. von Keyserlingk and S. L. Sondhi, Phase structure of onedimensional interacting floquet systems. I. Abelian symmetryprotected topological phases, Phys. Rev. B 93, 245145 (2016).

[43] D. V. Else and C. Nayak, Classification of topological phases in periodically driven interacting systems, Phys. Rev. B 93, 201103 (2016).

[44] A. C. Potter, T. Morimoto, and A. Vishwanath, Classification of Interacting Topological Floquet Phases in One Dimension, Phys. Rev. X 6, 041001 (2016).

[45] R. Roy and F. Harper, Abelian Floquet symmetry-protected topological phases in one dimension, Phys. Rev. B 94, 125105 (2016).

[46] J. Maldacena, S. H. Shenker, and D. Stanford, A bound on chaos, J. High Energy Phys. 08 (2016) 106.

[47] J. Berges, Sz. Borsányi, and C. Wetterich, Prethermalization, Phys. Rev. Lett. 93, 142002 (2004).

[48] M. Moeckel and S. Kehrein, Interaction Quench in the Hubbard Model, Phys. Rev. Lett. 100, 175702 (2008).

[49] J. Zhang, G. Pagano, P. W. Hess, A. Kyprianidis, P. Becker, H. Kaplan, A. V. Gorshkov, Z.-X. Gong, and C. Monroe, Observation of a many-body dynamical phase transition with a 53-qubit quantum simulator, Nature 551, 601 (2017). 